\section{Photoacoustic imaging of lacZ gene expression in vivo}

\author{
Li Li, ${ }^{a,+}$ Roger J. Zemp, ${ }^{a,+}$ Gina Lungu, ${ }^{b}$ George Stoica, \\ and Lihong V. Wang ${ }^{a}, *$ \\ ${ }^{a}$ Washington University in St. Louis, Optical Imaging \\ Laboratory, Department of Biomedical Engineering, St. Louis, \\ Missouri 63130 \\ ${ }^{\mathrm{b}}$ Texas A\&M University, Department of Pathobiology, \\ College Station, Texas 77840
}

Abstract. In the postgenomic era, imaging techniques are playing an important role in visualizing gene expression in vivo. This work represents the first demonstration of photoacoustic tomography (PAT) for reporter gene imaging. Rats inoculated with $9 \mathrm{~L} / \mathrm{lac} Z$ gliosarcoma tumor cells are imaged with PAT before and after injection of X-gal, a colorimetric assay for the lacZ-encoded enzyme $\beta$-galactosidase. Using far-red optical illumination, the genetically tagged tumors in rats are clearly visualized by PAT following the assay. The spatial resolution is quantified to be less than $400 \mu \mathrm{m}$, while 500-nM-level sensitivity is demonstrated. With the future development of new absorption-based reporter gene systems, it is anticipated that photoacoustic technology will provide a valuable tool for molecular imaging research. () 2007 Society of Photo-Optical Instrumentation Engineers. [DOI: 10.1117/1.2717531]

Keywords: photoacoustic tomography; gene expression; molecular imaging; lacZ; reporter gene.

Paper 06223LRR received Aug. 15, 2006; revised manuscript received Jan. 14, 2007; accepted for publication Jan. 30, 2007; published online Apr. 3, 2007.

Molecular imaging techniques are now being developed to visualize biochemistry and function to complement anatomical imaging techniques. In gene expression imaging, an exogenous "reporter gene" is usually incorporated into the genome of cell lines or the animal model of interest in such a way that its expression is under the control of some desired promoter or enhancer. By using a ubiquitously strong promoter, cells can be genetically tagged for in-vivo tracking. The expression products of reporter genes provide required contrast for an imaging modality either directly or indirectly via some assay. Because many diseases involve genetic disorders, either inherited or originating from environmental stress, visualization of gene expression may significantly impact the future of medicine.

Many widely used medical imaging modalities, such as positron emission tomography (PET), single photon emission tomography (SPECT), and magnetic resonance imaging (MRI), have been used to image gene expression in vivo. ${ }^{1}$ Recently, optical methods are attracting increasing interest because of various optical contrast mechanisms, minimized background noise, high sensitivity, use of nonionizing radia-

\footnotetext{
${ }^{\dagger}$ These authors contributed equally.
}

*E-mail: lhwang@biomed.wustl.edu tion, low cost, and ease of operation. ${ }^{2}$ While in-vivo optical microscopy offers micron-scale resolution, the applicability is primarily limited to submillimeter imaging depths in the dermis, where photon transportation is sufficiently ballistic. Planar fluorescence imaging technology using reporter genes such as green fluorescent protein enables whole body imaging of small animals, although at low spatial resolution due to multiple scattering of light. Bioluminescence imaging using the luciferase has seen great success in molecular imaging. It eliminates the problem of endogenous fluorescence encountered in planar fluorescence imaging; however, again it suffers from low spatial resolution of deep structures. Fluorescence tomography is emerging as a powerful molecular imaging tool, ${ }^{3}$ with submillimeter spatial resolution possible; ${ }^{4}$ however, the reconstruction procedures are also highly ill posed and dependent on optical diffusion theory, which breaks down within a few transport-mean-free paths, often a dimension of interest for small animals.

In this work, as well as our conference report, ${ }^{5}$ we demonstrate, for the first time to our knowledge, photoacoustic imaging as a promising candidate for imaging gene expression in vivo by exploiting a new contrast mechanism-optical absorption. Photoacoustic waves are generated by inducing thermo-elastic expansion in optically absorbing structures irradiated by pulsed laser light. In photoacoustic tomography (PAT), the photoacoustic waves are detected with ultrasonic transducers at various positions surrounding the object. The absorbing structures are then mapped into the image space through reconstruction. Image contrast in PAT is due to optical absorption, yet spatial resolution is based on ultrasonic detection and is not limited by optical scattering or reconstruction regularization. Photoacoustic spatial resolution can approach the ultrasonic diffraction limit, which is a fraction of a millimeter for diagnostic-frequency ultrasound. This resolution has been demonstrated up to several centimeters in tissue-mimicking phantoms. ${ }^{6-8}$ PAT has seen great success in small animal imaging during its short history. ${ }^{9,10}$ In this work, we demonstrate $400-\mu \mathrm{m}$ spatial resolution subdermal photoacoustic imaging of gene expression, a resolution scale finer than the state-of-the-art fluorescence tomography. ${ }^{4}$ While ultrasonic transducer bandwidth may be scaled to obtain even higher resolutions, the key emphasis in this work is to demonstrate the feasibility of using photoacoustic technology to visualize optical absorption as a new contrast mechanism for subdermal gene expression imaging.

We chose to image the expression of lacZ, one of the most widely used reporter genes. The lacZ gene encodes $\beta$-galactosidase, an E. coli enzyme responsible for lactose metabolism. We used the sensitive chromogenic assay 5-bromo-4-chloro-3-indolyl- $\beta$-D-galactoside (X-gal), an optically transparent lactose-like substrate, which yields a stable dark blue product following the cleavage of the glycosidic linkage by $\beta$-galactosidase. We demonstrate that PAT is sensitive to the increase in optical absorption due to this assay. Few other methods for imaging lacZ expression have been reported. One MRI approach used a $\beta$-galactosidase-cleavable paramagnetic contrast agent, which was introduced into a $\mathrm{Xe}$ nopus embryo at the two-cell stage. ${ }^{11}$ Recently, planar fluorescence imaging was used to image the fluorescent prod-

1083-3668/2007/12(2)/020504/3/\$25.00 @ 2007 SPIE 


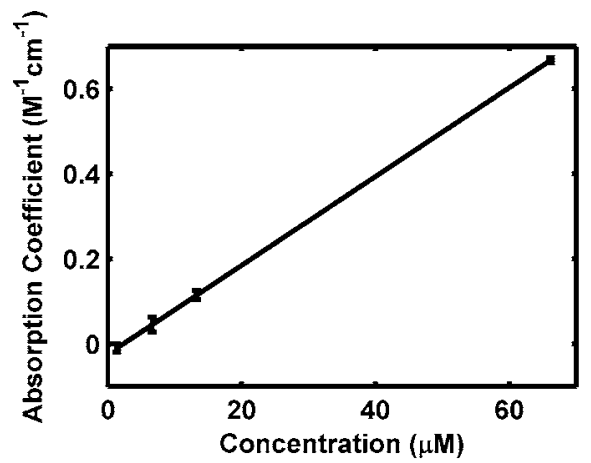

(a)

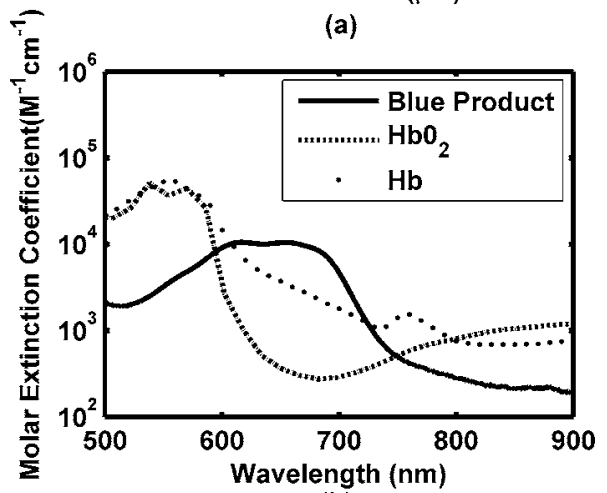

(b)

Fig. 1 (a) Linear fitting of absorbance versus concentration of the blue product at $650 \mathrm{~nm}$. (b) Comparison of absorption spectra for the blue product, $\mathrm{HbO}_{2}$, and $\mathrm{Hb}$.

uct from 9H-(1,3-dichloro-9,9-dimethylacridin-2-one-7-yl) $\beta$-D-galactopyranoside (DDAOG) cleaved by $\beta$-galactosidase. $^{12}$

We first measured the molar extinction spectrum of the blue product to determine an appropriate illumination wavelength. Concentrated $\beta$-galactosidase solution was obtained from the lysate of $9 \mathrm{~L} / \mathrm{lacZ}$ cells and then divided equally into five cuvettes. $20 \mu \mathrm{l}$ of X-gal solutions with different concentrations were added to the first four cuvettes, while $20 \mu \mathrm{l}$ of water was added to the last cuvette as a baseline. After given enough time for the reaction, these solutions were measured for the absorbance using a spectrometer (USB2000, Ocean Optics, Dunedin, FL). Assuming all the X-gal molecules were cleaved, we calculated the concentrations of the blue product. The assumption was verified by observing that the absorbance and concentration of the blue product at every single wavelength followed a linear relationship [Fig. 1(a)]. Molar extinction coefficients were obtained from the slope of Fig. 1(a). In Fig. 1(b), we compared the molar extinction spectrum of the blue product with those of the main biological absorbers: oxyhemoglobin $\left(\mathrm{HbO}_{2}\right)$ and deoxyhemoglobin $(\mathrm{Hb})$. We chose far-red light at $650 \mathrm{~nm}$, where the contrast between the absorption of the blue product over the background absorption from hemoglobin is high. Illumination at this wavelength can penetrate deeply into tissue.

A diagram of our PAT system is shown in Fig. 2(a). A dye laser pumped by a Q-switched Nd:YAG laser, emitted light at $650 \mathrm{~nm}$. Before reaching the animal, the light was broadened by a concave lens and a ground glass. The illumination was measured to be $\sim 5 \mathrm{~mJ} / \mathrm{cm}^{2}$ near the skin. An ultrasonic transducer with a center frequency of $2.25 \mathrm{MHz}$ was me-

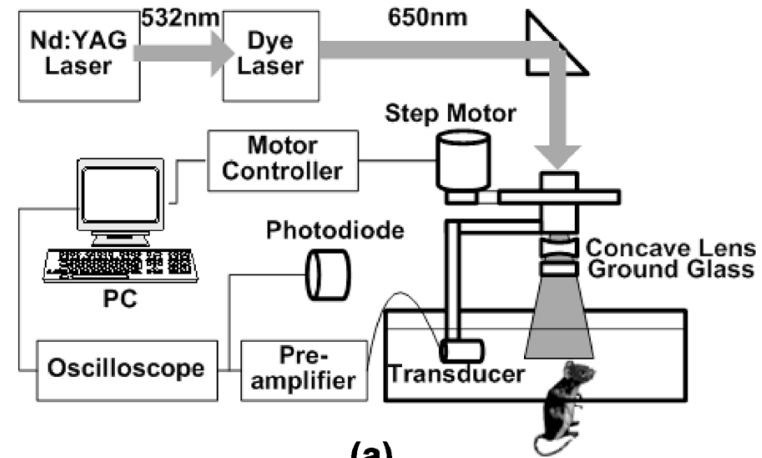

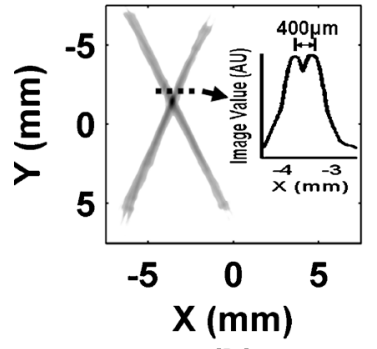

(b) (a)

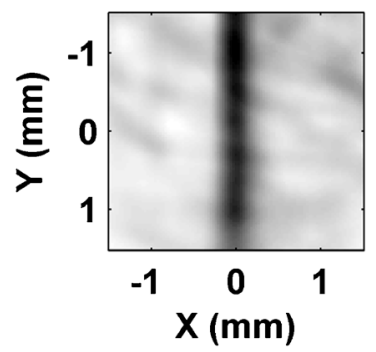

(c)
Fig. 2 PAT setup and experiments quantifying the system's performance. (a) Schematic of PAT system setup. (b) PAT image of two crossed hairs embedded in gelatin. The inset shows a 1-D profile of the dashed line, where two hairs are separated by $400 \mu \mathrm{m}$. (c) PAT image of a tube of $5.4-\mu \mathrm{M}$ blue product embedded $5 \mathrm{~mm}$ deep in chicken tissue.

chanically scanned along a circle with 240 steps. Signals were amplified, digitized, and averaged at each scan position. Signals from a photodiode were used to compensate for fluctuations in the illuminating energy. Images were reconstructed using a modified back-projection algorithm.

Approximately 5 million 9L/lacZ gliosarcoma tumor cells (American Type Culture Collection, Manassas, VA) were inoculated subcutaneously under the scalps of Sprague-Dawley rats. The tumors were left to grow until a small bump was present. Hair was removed before imaging. Animals were kept under anesthesia and their blood oxygenation and heart rates were monitored during experiments. $25 \mu \mathrm{l}$ of $\mathrm{X}$-gal solution $(20 \mathrm{mg} / \mathrm{ml}$, Fermentas, Hanover, MD) was injected subcutaneously near the tumor. The same amount of X-gal was injected in a nontumor location in the symmetric position on the contralateral side as a control. Two separate PAT images were taken before and after injection.

Figure 3(a) shows the PAT image prior to X-gal injection. While the median fissure and some cortical vessels are visible, the tumor is not. X-gal was injected at points indicated by arrows in Fig. 3(c). Following the assay, the tumor is clearly seen in Fig. 3(b). No signal up-regulation was observed at the right-side injection point, which served as a negative control. Figure 3(c) shows the photograph of the rat head following $\mathrm{X}$-gal injection and just prior to imaging. Although a small bump is present, the exact shape of the tumor and distribution of lacZ expression was unknown. Figure 3(d) shows the underside of the dissected scalp in the rectangular region indicated in Fig. 3(c), showing the X-gal stained tumor, which is dark blue in color. 


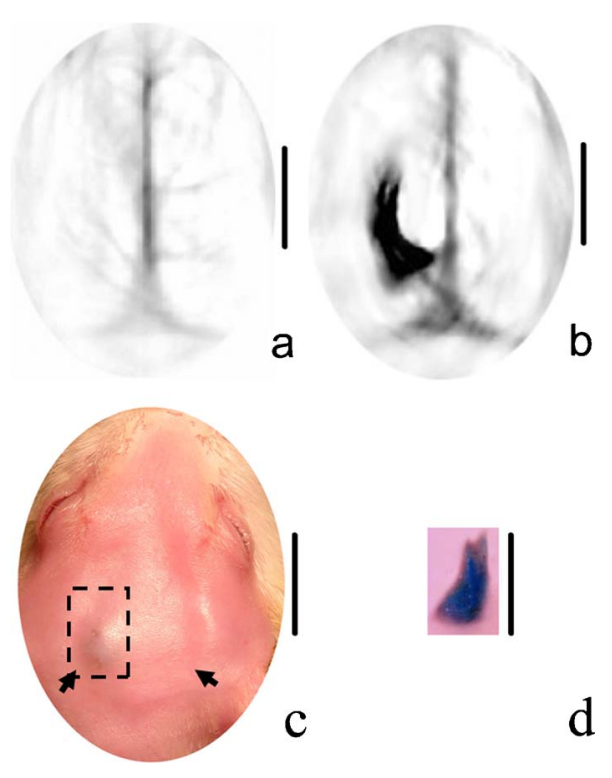

Fig. 3 Results from photoacoustic tomography at $650 \mathrm{~nm}$. (a) and (b) Photoacoustic images before and after the injection of X-gal. (c) Photograph of the rat's head prior to second scan. The two arrows indicate the position of injection of X-gal. (d) Photograph of the underside of the rat's scalp in the rectangular region in (c), excised after sacrificing the animal. The line scale represents $5 \mathrm{~mm}$.

The in-plane spatial resolution was estimated by imaging a crossed-hair phantom, shown in Fig. 2(b). The two hairs become clearly distinct at the $400-\mu \mathrm{m}$ separation, which means the resolution of our system is less than this. To estimate the molar sensitivity, we imaged a $250-\mu \mathrm{m}$ inner diameter tube, transparent to light and ultrasound, filled with a solution of blue product at a concentration of $5.4 \mu \mathrm{M}$ and embedded $5 \mathrm{~mm}$ deep in chicken tissue [Fig. 2(c)]. The region with blue product was estimated to be 3.1 times stronger than the background with a contrast-to-noise ratio (CNR) of 10.5. Hence, the minimum detectable concentration of blue product (with $\mathrm{CNR}=1$ ) was less than $515 \mathrm{nM}$. Compared with other subdermal imaging technologies with submillimeter resolution, this technique has a minimum detectable concentration less than the submillimolar levels typically required in MRI, ${ }^{1}$ but higher than the $100 \mathrm{fM}$ levels possible with fluorescence tomography. ${ }^{4}$

Note that the lacZ reporter gene also possesses an intrinsic signal-amplification mechanism. As an enzyme, a single $\beta$-galactosidase molecule can cleave multiple $\mathrm{X}$-gal molecules to produce a large number of blue product molecules. Hence our calculated minimum detectable concentration pertains to the blue product, while the actual levels of $\beta$-galactosidase, the real expression product, may be significantly lower. Multiwavelength techniques can be applied to push this limit of sensitivity even further by exploiting the spectral information to recognize specific absorbers from the background.

It took us $25 \mathrm{~min}$ to acquire one frame, due to the slow $10-\mathrm{Hz}$ pulse repetition rate (PRF) of the laser and the timeconsuming mechanical scanning of the single-element ultra- sonic detector. Scan time can be greatly improved with high PRF lasers and ultrasonic phased arrays.

There are some limitations for in-vivo applications of $\mathrm{X}$-gal. It can cause skin irritation occasionally, and the in-vivo dynamics of the reaction is poorly understood. Systemic delivery through the tail vein seems inefficient in our animal model. Given the enormous number of pigments existing in nature, however, future development of absorption-based reporter gene systems may provide new approaches for PAT of gene expression in vivo. An ideal reporter gene system should have a simple reaction mechanism with minimized requirements for delivery, and should generate highly absorbing products in the near-infrared region of the optical spectrum.

In summary, we demonstrate the feasibility of imaging gene expression by photoacoustic tomography for the first time. The concept of using reporter genes that modulate optical absorption as an image contrast mechanism represents a new paradigm for gene expression imaging. We believe that the development of new absorption-based reporter gene systems may provide new opportunities in molecular imaging and basic science research.

\section{Acknowledgment}

We are grateful to Sergio Similache and Ovidiu Craciun for assistance with animal handling. This research is funded in part by the NIH grants R01 NS46214 (BRP) and R01 EB000712.

\section{References}

1. T. F. Massoud and S. S. Gambhir, "Molecular imaging in living subjects: seeing fundamental biological processes in a new light," Genes Dev. 17, 545-580 (2003).

2. R. Weissleder and V. Ntziachristos, "Shedding light onto live molecular targets," Nat. Med. 9, 123-128 (2003).

3. V. Ntziachristos, C. Tung, C. Bremer, and R. Weissleder, "Fluorescence molecular tomography resolves protease activity in vivo," Nat. Med. 8, 757-761 (2002).

4. E. E. Graves, J. Ripoll, R. Weissleder, and V. Ntziachristos, "A submillimeter resolution fluorescence molecular imaging system for small animal imaging," Med. Phys. 30, 901-911 (2003).

5. L. Li, R. J. Zemp, G. Lungu, G. Stoica, and L. H. Wang, "Imaging of gene expression in vivo with photoacoustic tomography," Proc. SPIE 6086, 608608 (2006).

6. C. G. A. Hoelen, F. F. M. de Mul, R. Pongers, and A. Dekker, "Threedimensional photoacoustic imaging of blood vessels in tissue," Opt. Lett. 23, 648-650 (1998).

7. R. O. Esenaliev, A. A. Karabutov, and A. A. Oraevsky, "Sensitivity of laser opto-acoustic imaging in detection of small deeply embedded tumors," IEEE J. Sel. Top. Quantum Electron. 5, 981-988 (1999).

8. G. Ku and L. V. Wang, "Deeply penetrating photoacoustic tomography in biological tissues enhanced with an optical contrast agent," Opt. Lett. 30, 507-509 (2005).

9. X. Wang, Y. Pang, G. Ku, X. Xie, G. Stoica, and L. V. Wang, "Noninvasive laser-induced photoacoustic tomography for structural and functional imaging of the brain in vivo," Nat. Biotechnol. 21, 803806 (2003).

10. R. A. Kruger, W. L. Kiser, D. R. Reinecke, G. A. Kruger, and K. D. Miller, "Thermoacoustic optical molecular imaging of small animals," Mol. Imaging 2, 113-123 (2003).

11. A. Y. Louie, M. M. Huber, and E. T. Ahrens, "In vivo visualization of gene expression using magnetic resonance imaging," Nat. Biotechnol. 18, 321-325 (2000).

12. C. Tung, Q. Zeng, K. Shah, D. E. Kim, D. Schellingerhout, and R. Weissleder, "In vivo imaging of $\beta$-galactosidase activity using far red fluorescent switch," Cancer Res. 64, 1579-1583 (2004). 